\title{
Differences in blood flow to uterine segments and placentae in relation to sex, intrauterine location and side in pregnant rats
}

\author{
M. D. Even ${ }^{1}$, M. H. Laughlin ${ }^{2}$, G. F. Krause ${ }^{3}$ and F. S. vom Saal ${ }^{1 *}$ \\ ${ }^{1} J o h n$ M. Dalton Research Center, Division of Biological Sciences; ${ }^{2}$ Department of Veterinary Biomedical \\ Sciences; and ${ }^{3}$ Department of Statistics, University of Missouri-Columbia, Columbia, MO 65211, USA
}

\begin{abstract}
The effects of location within the left or right uterine horn, position within each uterine horn, and fetal sex on fetal bodymass, blood flow to individual uterine segments associated with fetuses, and blood flow to the maternal portion of the placenta were investigated in rats. Sprague-Dawley rats were anaesthetized on day 5, 10, 15, 20, 21 or 22 of pregnancy, and radioactive microspheres with diameters of $15 \mathrm{~mm}$ were injected via a left ventricular cannula to measure blood flow to tissues. Tissues were weighed wet, and the rate of blood flow, corrected for wet mass ( $\mathrm{ml} \mathrm{min}{ }^{-1} \mathrm{~g}^{-1}$ tissue), was calculated. Microspheres were not detected in fetuses, suggesting that they did not pass from maternal into fetal blood. Uterine blood flow was greater at the cervical and ovarian ends than in the middle of the uterus; on day 15 the rate of blood flow at the cervical and ovarian ends of each uterine horn was over twice that in the middle. The blood flow to the right uterine horn was greater than to the left horn. Blood flow to placentae increased dramatically between day 15 and day 20 . There were marked differences in architecture between the uterine artery feeding the ovarian end of the right and left uterine horn, and blood flow to placentae located at the ovarian end of the right uterine horn was greater than to placentae in the same location in the left uterine horn. The blood flow to placentae and fetal bodymass were greater for female than for male fetuses on day 20 , but on day 22 the reverse was observed. There was no effect of the number of fetuses in the same uterine horn on blood flow to the uterus or placentae, or on fetal or placental mass.
\end{abstract}

\section{Introduction}

In many litter-bearing species, there is a negative correlation between litter size and fetal mass. This effect of litter size has been shown to occur in guinea-pigs (Ibsen, 1928; Eckstein and McKeown, 1955), mice (McLaren and Michie, 1959; 1960; Healy et al., 1960; McLaren, 1965), rabbits (Hammond, 1944) and rats (Barr et al., 1969, 1970). A number of hypotheses have been put forward to explain the effect of intrauterine crowding on fetal size, including competition for a limited amount of nutrients, space or blood.

Rat and mice have a duplex uterus (two separate uterine horns), with a loop artery feeding each uterine horn (Fig. 1). It is predicted that blood enters the uterine loop artery in rats and other rodents from both directions; thus, the placentae in the middle of a uterine horn probably experience the lowest perfusion pressure (McLaren and Michie, 1960). Evidence that the smallest fetuses are located in the middle region of a uterine horn supports this hypothesis in mice (McLaren and Michie, 1960), guinea-pigs (Eckstein et al., 1955) and rabbits (Bruce and Abdul-Karim, 1973). Presumably, the more placentae that are fed by a uterine artery, the lower the pressure would be within that aretery; a decrease in blood pressure would also be

*Correspondence.

Revised manuscript received 3 June 1994. associated with a decrease in the amount of oxygen and other nutrients supplied to more distal placentae and, ultimately, to the fetuses nourished by these placentae. Thus, insufficient blood flow could account for the local effect on fetal mass at birth seen within crowded uterine horns in mice (McLaren and Michie, 1960). In contrast, although the uterine vasculature of rats appears similar to that of mice, there have been reports that in rats the smallest fetuses are found at either the ovarian or cervical ends of a crowded uterine horn (Ward et al, 1977; Norman and Bruce, 1979a, b).

In addition to local effects exerted by fetuses upon each other within a uterine hom, in rats and mice, there is evidence for effects of number of fetuses on fetal growth owing to systemic effects (i.e. exerted by fetuses located in the opposite uterine horn; Eckstein and McKeown, 1955; McLaren and Michie, 1960). It is possible that systemic effects are due to the blood flow in one uterine artery influencing the blood flow in the opposite uterine artery, with the result that a crowded uterine horn could lead to a retardation of growth of fetuses in the contralateral uterine horn. As described above, the greatest effect would be on those fetuses in the contralateral uterine horn that receive the lowest placental perfusion pressure.

In this study, we examined the relationship between fetal bodymass and both uterine and placental blood flow on days 5 , $10,15,20,21$ and 22 of pregnancy in the rat. Blood flow was determined using radioactive microspheres that lodge 


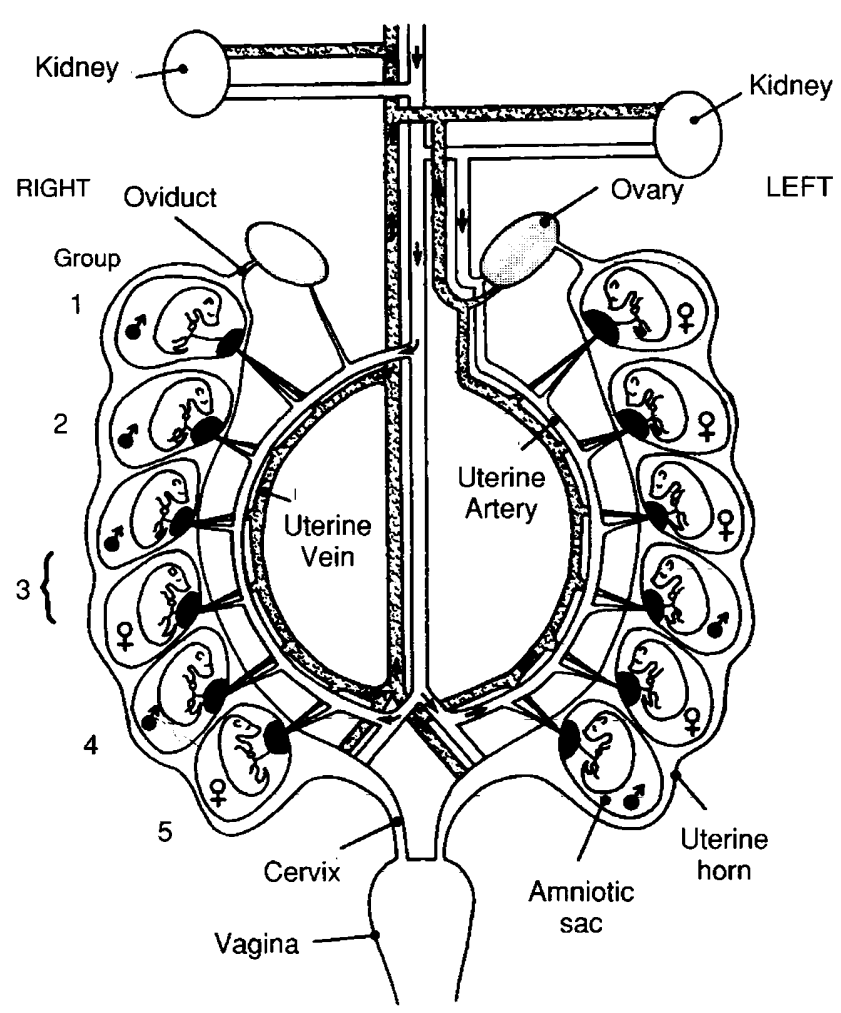

Fig. 1. The uterine horns and the uterine loop artery and vein that feed each horn in a pregnant rat near the end of pregnancy. Fetuses, placentae and segments of uterine tissue associated with each fetus were identified as belonging to one of five groups based on their position relative to the ovary and cervix: (1) next to the ovary; (2) second position away from the ovary; (3) positions $1-3$ in the middle region of the uterine horn; (4) second position away from the cervix; and (5) next to the cervix. Arrows within the vessels identify the predicted direction of blood flow at different points in the vessels, based on results of the experiment reported here for arterial flow and elsewhere for both arterial and venous flow (from vom Saal and Dhar, 1992 with permission).

in capillaries as a function of the rate of blood flow to the tissue.

\section{Materials and Methods}

\section{Animals}

Harlan Sprague-Dawley rats were housed individually at $25 \pm 2^{\circ} \mathrm{C}$ under photoperiod of $12 \mathrm{~h}$ light: $12 \mathrm{~h}$ dark, with lights on at $10: 00 \mathrm{~h}$ in $45 \mathrm{~cm} \times 25 \mathrm{~cm} \times 20 \mathrm{~cm}$ polyethylene cages on Aspen bedding. Females were mated when they were 5-6 months old, and the day on which spermatozoa were observed in the female tract was taken as day 0 of pregnancy. A $10 \%$ increase in mass 10 days later was taken as evidence of pregnancy.

\section{Determination of shunting of microspheres}

On day 21 of pregnancy, when maximum dilation of uterine vessels occurs (Fig. 2; H. Bruce, personal communication), we

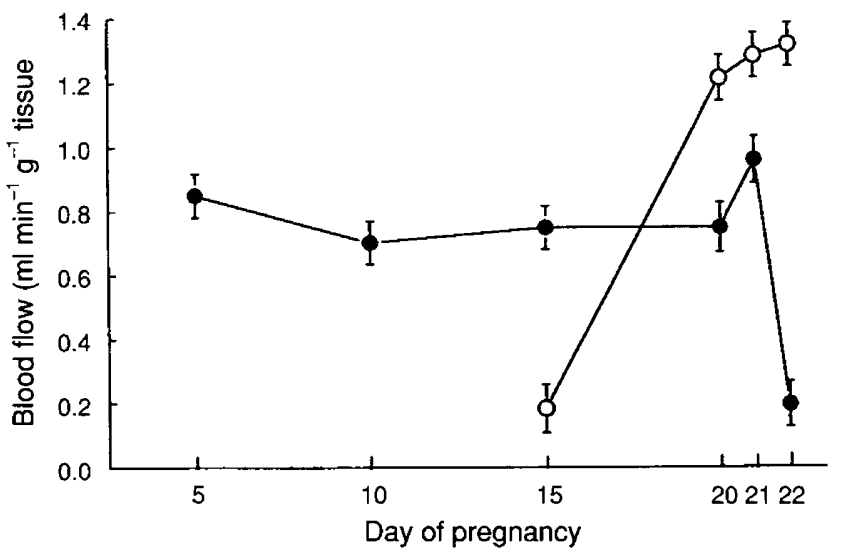

Fig. 2. Mean ( \pm SEM) blood flow (corrected for wet mass) to placentae $(O)$ and uterine segments $(\bullet)$ associated with fetuses in pregnant rats. The blood flow to placentae was not determined before day 15 of pregnancy.

estimated the degree of arteriovenous shunting that occurs across the utero-placental circulation following intracarotid injection of radiolabelled microspheres $15 \mathrm{~mm}$ in diameter. The isotopes used were ${ }^{46} \mathrm{Sc},{ }^{151} \mathrm{Ce},{ }^{113} \mathrm{Sn}$ and ${ }^{95} \mathrm{Nb}$ (DuPont NEN, Boston, MA); the isotope used did not influence the results. Eight pregnant females were anaesthetized with an i.p. injection of $35 \mathrm{mg}$ sodium pentobarbital $\mathrm{kg}^{-1}$. A polyethylene catheter (size: PE-50) for infusion of microspheres was filled with a solution of $1 \%$ heparizined saline. An incision was made in the neck, and the catheter was threaded through the right carotid artery into the left ventricle. A similar catheter was placed into the tail artery for reference blood withdrawals.

In the experiment, reference blood samples were collected only from a catheter inserted into the tail artery. However, in this pilot study, females also had a catheter (size: PE-100) placed into the inferior vena cava via the right jugular vein, at a point just superior to the junction of the renal veins with the inferior vena cava. This catheter was used to determine the amount of microsphere shunting across the uterine arterial system into the uterine venous system. The only vessels feeding into the vena cava at this location, the iliofemoral and renal veins, were ligated so that only blood from the uterine vascular bed could enter the vena cava. In addition, umbilical tape was tightly tied around each hind leg to reduce blood flow through the legs as much as possible. The percentage of tail arterial reference counts found in the uterine venous outflow was used to estimate the amount of microsphere shunting that occurred in the uterine vasculature. All catheters were periodically flushed with heparinized saline to prevent clotting. The animals were infused with a $0.2 \mathrm{ml}$ suspension containing microspheres (15 $\mathrm{mm}$ in diameter) in a $10 \%$ dextran solution containing $0.1 \%$ Tween-80. Approximately 500000 microspheres were infused. Withdrawals from reference cannulae were made using pumps at a rate of $0.5 \mathrm{ml} \mathrm{min}{ }^{-1}$ for the first 5 min after microsphere injection, after which the animal was killed by opening the pleural cavity.

Minimal shunting $(5.2 \pm 0.8 \%)$ occurred across the uterine vasculature, which may reflect erosion of the maternal vascular bed apposed to the fetal placenta; if so, the blood flow to placentae may be slightly underestimated. No radioactivity was 
detected in fetuses, suggesting the microspheres did not cross from the maternal blood in the maternal portion of the placenta into the fetal blood in the fetal portion of the placenta (rats have a haemochorial placenta). When the blood flow to placentae was corrected for placental mass (referred to as the relative blood flow to placentae), measurement of placental mass included the maternal component as well as the fetal component of the placenta, which probably did not contain microspheres.

\section{Blood flow to uterine segments and placentae throughout pregnancy}

Blood flow to the maternal portion of the placenta and uterine segments associated with fetuses was examined between $14: 00$ and $17: 00 \mathrm{~h}$ on days $5,10,15,20,21$ or 22 of pregnancy in 5-7 pregnant females per day. Hind legs were not tied, and shunting across the uterine vasculature was not estimated because of the low percentage of shunting that occurred in the pilot study. Parturition in $75 \%$ of rats in this colony occurs during day 22 of pregnancy, and only females that had not started delivering pups by $14: 00 \mathrm{~h}$ on day $22(4 \mathrm{~h}$ after the onset of the light phase) were used.

Individual sites of implantation in females were identified on day 5 of pregnancy by injecting $0.2 \mathrm{ml}$ of Evan's blue dye via the left ventricular catheter immediately after the infusion of microspheres. After the animal had been killed, the uterus was cut into individual segments to count the number of blue bands, which indicate the points where implantation had occurred. Fetal and placental mass and placental blood flow were not measured on day 5 or day 10 of pregnancy.

Beginning on day 15 of pregnancy, mass and blood flow were determined for each placenta as well as for each uterine segment, and fetal masses were also recorded. On days 20, 21 and 22 of pregnancy, fetal sex was determined by measuring the length of the space separating the anus and genital papilla; fetal sex was not determined before day 20. The anogenital distance unambiguously distinguishes males and females near the end of pregnancy (Clemens et al., 1978).

The animals were killed 5 min after injecting the microspheres. Fetuses, placentae, uterine segments associated with individual fetuses (cut midway between two fetuses), and maternal kidneys were removed; wet masses were recorded, and tissues were dried and counted in a Tracor gamma counter using Tracor software.

\section{Statistical analyses}

Data were analysed by analysis of variance (ANOVA) using the general linear model (GLM) in the Statistical Analysis System (SAS). Planned comparisons were made using the LS means test in SAS. Data were also analysed by analysis of co-variance (ANCOVA), and two variables were examined for their relationship to the other dependent measures: (i) the number of pups in the same uterine horn in which the measurements were made; and (ii) the number of pups in the contralateral uterine horn. Pearson's correlation coefficients were also calculated to determine whether the number of pups in the same or opposite uterine horn were positively or negatively correlated with other measures.
For purposes of analysis, fetuses, placentae and uterine segments were assigned to one of five groups according to their position within each uterine hom relative to the ovary or cervix (Fig. I): (1) next to the ovary; (2) second position away from the ovary; (3) positions $1-3$ in the middle region of the uterine horn; (4) second position away from the cervix; and (5) next to the cervix.

If there were six or more fetuses in a single uterine horn, then the 2-3 fetuses in the middle (as well as uterine segments and placentae associated with them) were included in group 3. If there were either eight or ten fetuses in a uterine horn, then only the data from the middle two fetuses were included in group 3, and if there were seven, nine or II fetuses per horn, then the data from the middle three fetuses were included in group 3. In uterine horns containing less than five fetuses (this occurred in eight of 72 uterine horns examined), there were no data for group 3; one objective of conducting the ANCOVA was to identify whether the results were biased by an underrepresentation of pups in this group. However, the analyses showed that the few cases in which there were fewer than five fetuses in a uterine horn had no impact on the overall results.

The radioactivity found in the tissues was compared with radioactivity from the reference withdrawal sample collected from the tail artery, and the ratio was used to calculate the rate of blood flow. The rate of blood flow (in $\mathrm{ml} \mathrm{min}^{-1}$ ) to the tissues of interest was calculated according to the formula:

$$
\text { Blood flow }=Q_{\text {ref }} \times\left(N_{\text {tis }} \text { per } N_{\text {ref }}\right) \text {, }
$$

where $Q_{\text {ref }}$ is the rate of withdrawal of the reference sample in $\mathrm{ml} \min ^{-1}\left(0.5 \mathrm{ml} \mathrm{min}^{-1}\right), N_{\text {tis }}$ is the total radioactivity in counts per minute (c.p.m.) in the tissue, and $N_{\text {ref }}$ is the total radioactivity (c.p.m.) in the reference sample (Norjavaara $e t$ al., 1987). The blood flow to uterine segments and the maternal portion of placentae was expressed relative to tissue wet mass as $\mathrm{ml} \mathrm{min}^{-1} \mathrm{~g}^{-1}$ tissue, and was thus referred to as relative blood flow. The blood flow to the maternal placenta was also expressed as absolute $\left(\mathrm{ml} \mathrm{min}^{-1}\right)$ without being corrected for wet mass, since the results of our preliminary experiment suggested that microspheres had not entered the fetal portion of the placenta, which is included in placental mass. Results for both absolute and relative blood flow to placentae are therefore presented.

Both maternal kidneys were weighed and analysed for radioactivity. A large difference in flow to the right compared with the left kidney indicates improper mixing of microspheres. Relative blood flow to the left and right kidneys differed by less than $20 \%$ for each animal in our study: $3.33 \pm 0.23$ (mean $\pm \mathrm{SEM}$ ) $\mathrm{ml} \mathrm{min}^{-1} \mathrm{~g}^{-1}$ tissue for the left kidney; and $3.40 \pm 0.24$ (mean \pm SEM) $\mathrm{ml} \mathrm{min}^{-1} \mathrm{~g}^{-1}$ tissue for the right kidney $\left.\left(F_{1,69}\right)=0.06 ; P>0.1\right)$.

\section{Results}

\section{Uterine vascular anatomy}

In rats and other rodents, the two separate uterine horns are each fed by a single main loop artery and vein, which run in parallel. We observed that in Sprague-Dawley rats the vasculature of the left uterine horn was variable in its architecture, 
while the vasculature of the right uterine horn was very similar in all the animals examined. More specifically, the sections of the artery and vein leading to the ovarian end of the right uterine horn begin as major branches from the descending aorta and ascending vena cava, respectively; the ovarian artery and vein then branch off from the main loop uterine vessels. In contrast, the sections of the uterine artery and uterine vein feeding the ovarian end of the left uterine horn begin as branches of the renal artery and vein in most (but not all) animals; these vessels typically lead to the left ovary, and the actual loop uterine artery and uterine vein form an anastamosis with these vessels. However, in one pregnant rat (not used in the present study), we observed that the uterine loop artery and vein failed to anastamose with the rostral artery and vein leading to the left ovary; instead, each of these vessels formed a circle, looping back upon itself, at its rostral end. The caudal branches of the uterine loop artery and vein originate from the iliac artery and vein on both sides, as described in rats by $\mathrm{Del}$ Campo and Ginther (1972).

\section{Uterine blood flow}

Relative uterine blood flow ( $\mathrm{ml} \min ^{-1} \mathrm{~g}^{-1}$ tissue) differed $(P<0.001)$ as a function of day of pregnancy (Fig. 2). The greatest change occurred during the last day of pregnancy (day 22), when uterine blood flow dropped precipitously. Blood flow to uterine segments also differed significantly as a function of position of the uterine segment (Fig. 3). Ignoring day of pregnancy, both the ovarian (group 1) and cervical (group 5) sections of the uterus received a higher $(P<0.01)$ rate of blood flow than the middle section of the uterus (group 3), while the uterine segments comprising groups 2 and 4 did not differ significantly $(P=0.07-0.1)$ from groups 1,3 or 5 in their uterine blood flow. This effect was most pronounced on day 15 of pregnancy, at which time the rate of blood flow to uterine segments located at either end of a uterine hom was over two times greater than to segments located in the middle. In contrast, by day 21 of pregnancy, when the rate of fetal growth is highest, blood flow to the ovarian, middle and cervical segments of the uterus was similar due to an increase in blood flow to middle segments but not to ovarian and cervical segments.

Excluding data from day 22 owing to the dramatic drop in blood flow associated with parturition, the blood flow to the right uterine horn tended to be higher [by $21 \% ; 0.86 \pm 0.05 \mathrm{ml}$ $\min ^{-1} \mathrm{~g}^{-1}$ (mean $\left.\left.\pm \mathrm{SEM}\right)\right]$ than to the left uterine horn $\left(0.71 \pm 0.05 \mathrm{ml} \mathrm{min}^{-1} \mathrm{~g}^{-1} ; P=0.06\right)$. Blood flow to the right uterine horn was greater than blood flow to the left horn across all individual uterine segments, with right uterine segments in groups $1-5$ receiving $21 \%, 13 \%, 36 \%, 14 \%$ and $10 \%$, respectively, greater blood flow than the corresponding left segments. ANCOVA showed that the number of pups in either the same or opposite uterine horn had no significant effect on relative uterine blood flow.

\section{Blood flow to placentae and placental mass}

Relative blood flow to placentae. Relative placental blood flow ( $\mathrm{ml} \mathrm{min} \mathrm{m}^{-1} \mathrm{~g}^{-1}$ tissue) increased markedly between day

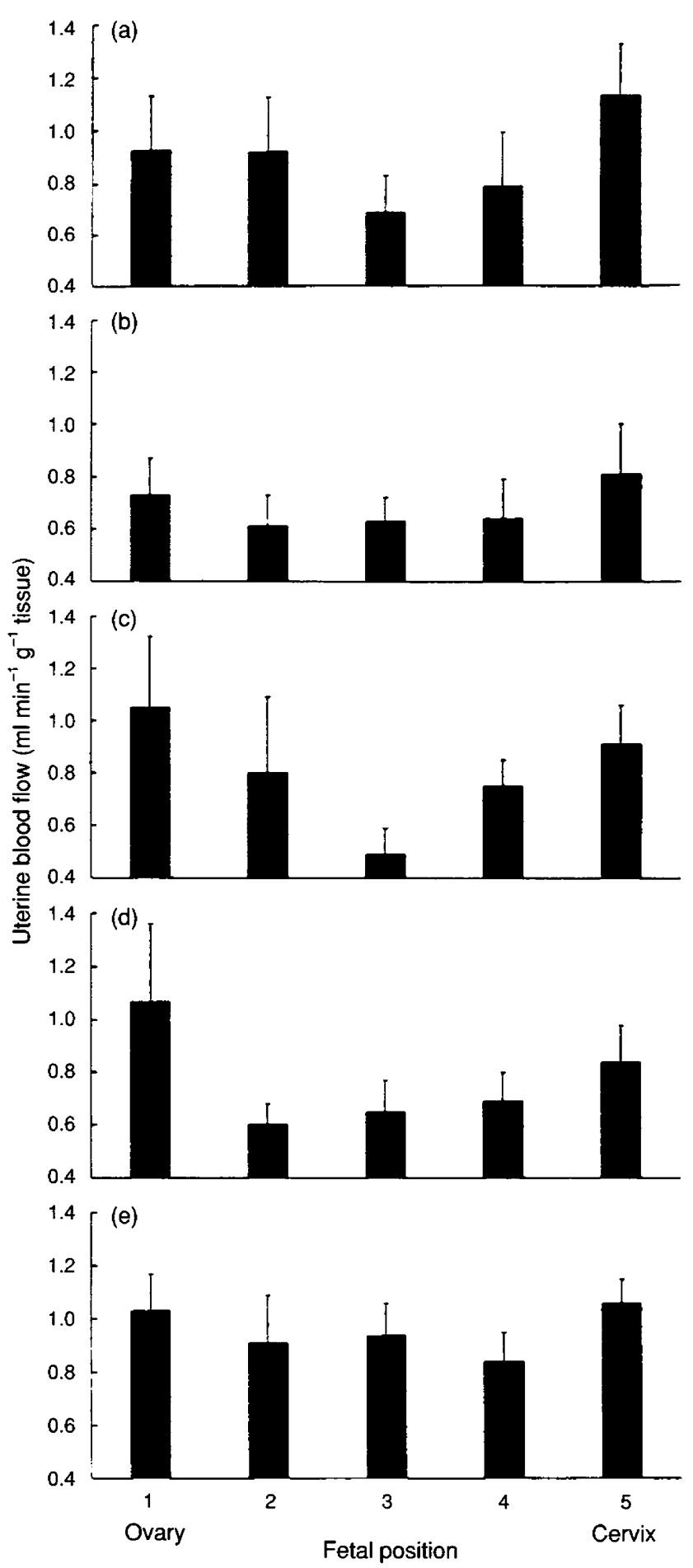

Fig. 3. Mean $(+$ SEM) blood flow to uterine segments (corrected for wet mass) associated with fetuses located at different positions [groups 1-5: (1) next to the ovary; (2) second position away from the ovary; (3) positions 1-3 in the middle region of the uterine horn; (4) second position away from the cervix; and (5) next to the cervix] within a uterine horn on (a) day 5, (b) day 10, (c) day 15, (d) day 20 and (e) day 21 of pregnancy in rats. (The data for day 22 are not included, since uterine blood flow was markedly reduced on this day; see Fig. 2.) 


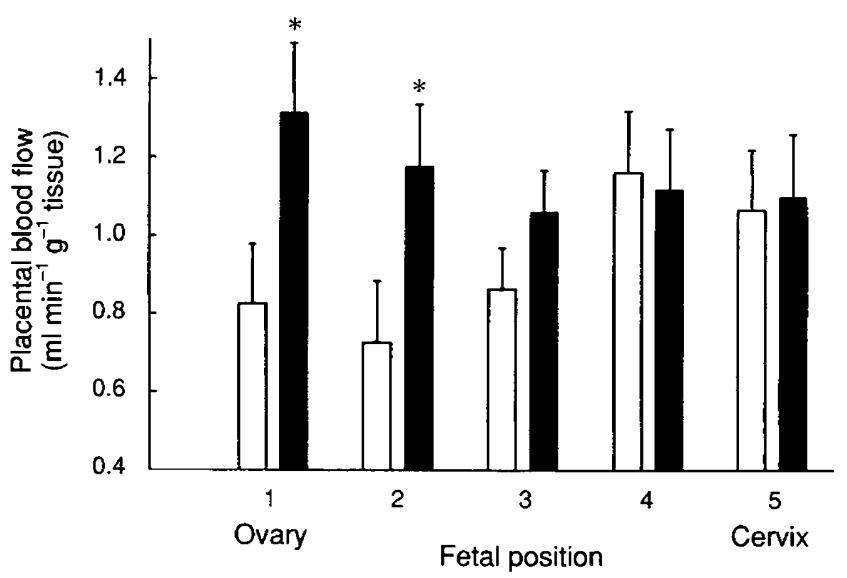

Fig. 4. Mean (+ SEM) blood flow to placentae (corrected for wet mass) associated with fetuses located at different positions [groups 1-5: (1) next to the ovary; (2) second position away from the ovary; (3) positions $1-3$ in the middle region of the uterine horn; (4) second position away from the cervix; and (5) next to the cervix] within the left $(\square)$ and right ( $\square$ ) uterine horns for placentae in rats collected on days $15,20,21$ and 22 of pregnancy. $* p<0.01$ indicates a significant difference between left and right uterine horns.

15 and day 20 of pregnancy and then remained constant up to day 22 (Fig. 2). The blood flow to placentae in the right uterine horn $\left(1.13 \pm 0.05 \mathrm{ml} \mathrm{min}^{-1} \mathrm{~g}^{-1}\right)$ was $37 \%$ greater $(P<0.01)$ than that to placentae in the left uterine horn $(0.92 \pm 0.05 \mathrm{ml}$ $\left.\min ^{-1} \mathrm{~g}^{-1}\right)$. Significant differences between group means in blood flow to placentae using the LS means test were observed only for placentae located at the ovarian end of the left uterine horn compared with the right uterine horn (groups 1 and 2; Fig. 4).

There was a significant interaction between fetal sex and day of pregnancy in terms of blood flow to placentae $(P<0.01)$. The placentae of female fetuses had a higher $(P<0.05)$ rate of blood flow than those of males on day 20 and 21 of pregnancy, while on day 22 the placentae of male fetuses had a higher $(P<0.05)$ rate of blood flow than those of females. Specifically, blood flow to the placentae of males increased between day 20 and day 22, while blood flow to placentae of females was similar between day 20 and day 21 and then decreased between day 21 and day 22 (Fig. 5). On each day that placental blood flow was examined, relative blood flow to uterine segments and to placentae were positively correlated $(r=0.54$, $0.44,0.47,0.36$ for days $15,20,21$ and 22 , respectively; $n=60-65$ per day; for all correlation coefficients, $P<0.01$ ).

When the data for days 20,21 and 22 were analysed by ANCOVA, the number of pups in the same horn did not account for a significant portion of the variance in the blood flow to placentae $(P>0.1)$. In contrast, the number of pups in the opposite horn accounted for a significant portion of the variance in blood flow to placentae $(P<0.001)$, although there was only a weak positive correlation (Pearson's correlation coefficient $=0.27, P<0.001$ ). All differences described using ANOVA remained statistically significant after ANCOVA.

Absolute blood flow to placentae. Results for absolute rate of blood flow to placentae (expressed as $\mathrm{ml} \mathrm{min}^{-1}$, and thus uncorrected for placental mass) were similar for all analyses to

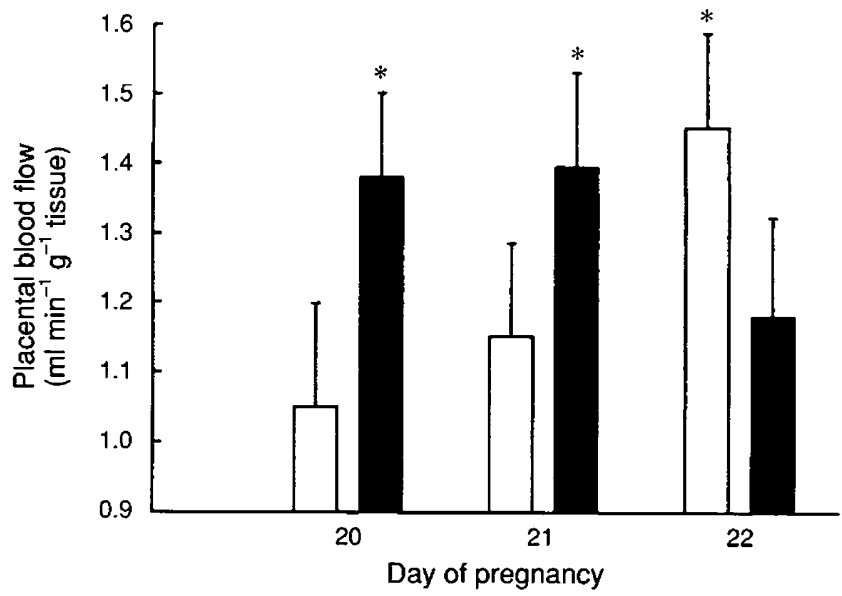

Fig. 5. Mean ( + SEM) blood flow to placentae (corrected for wet mass) associated with male $(\square)$ and female ( $\square$ ) fetuses for the last three days of pregnancy in rats. ${ }^{*} P<0.05$ indicates a significant difference between male and female fetuses.

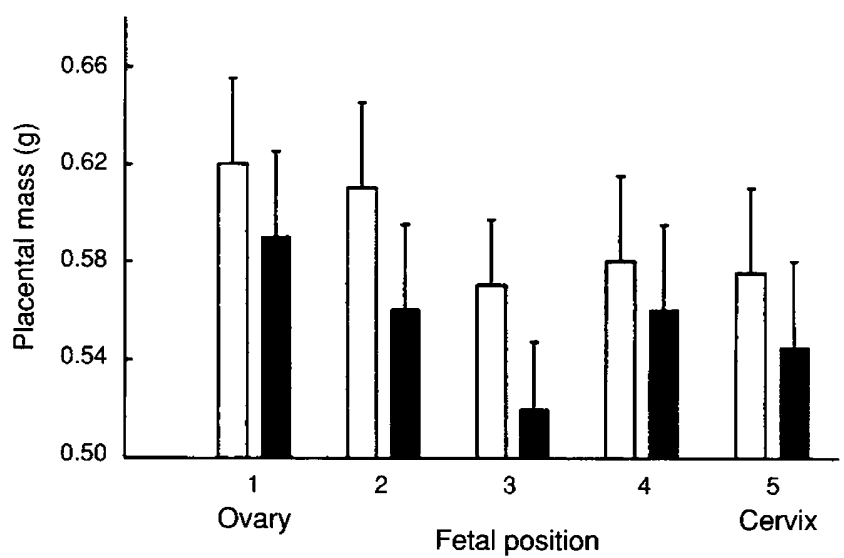

Fig. 6. Mean ( + SEM) mass of placentae located at different positions Igroups 1-5: (1) next to the ovary; (2) second position away from the ovary; (3) positions $1-3$ in the middle region of the uterine horn; (4) second position away from the cervix; and (5) next to the cervix] within the left $(\square)$ and right $(\square)$ uterine horns for placentae collected on days $15,20,21$ and 22 of pregnancy.

those described for relative blood flow to placentae, although individual $P$ values differed slightly. These findings show that the differences reported for the relative blood flow to placentae were not an artifact of correcting for differences in placental wet mass.

Placental mass. Placental mass increased $(P<0.01)$ between day $15(0.30 \pm 0.01 \mathrm{~g})$ and day $20(0.63 \pm 0.01 \mathrm{~g})$, and between day 20 and day $21(0.68 \pm 0.01 \mathrm{~g})$. It then decreased $(P<0.01)$ between day 21 and day $22(0.62 \pm 0.01 \mathrm{~g})$. Placental mass differed slightly but not significantly $(P=0.1)$ as a function of location in a uterine horn when data from days 15, 20,21 and 22 were combined (Fig. 6). A planned comparison was conducted based on the prediction that only groups 1 and 5 would differ from group 3. The LS means test showed that placentae located at the ovarian end of the uterine horns (group 1 ) were significantly $(P<0.01)$ heavier than placentae located in the 
middle region of the horns (group 3), but results from groups 3 and 5 did not differ significantly.

The general pattern for mean placental mass as a function of fetal position was similar for both the right and left uterine horns. ANOVA showed no significant difference in the mass of placentae located in the left compared with the right uterine horns, although placentae in the left uterine hom were slightly heavier $(P=0.1)$ than placentae in the right uterine horn. However, when the number of pups in the same $(P<0.01)$ or opposite $(P<0.05)$ uterine horn was used as the co-variate, ANCOVA revealed that the mass of placentae in the left uterine horn was significantly greater (by about $10 \%$ ) than that of placentae in the right uterine horn. This finding was unexpected, since blood flow to both the uterine tissue $(P=0.06)$ and placentae $(P<0.05)$ was greater for the right than for the left uterine hom. Placental mass did not differ significantly as a function of sex of the fetus; there was also no interaction between sex and day of pregnancy for placental mass $(P=0.5)$.

There was no significant correlation between relative uterine blood flow and placental mass on any day of pregnancy $(P>0.1)$. Absolute blood flow to placentae and placental mass were negatively correlated on day $20(r=-0.24, P=0.06)$, day $21(r=-0.30, P<0.05)$, day $22(r=-0.21, P=0.1)$. On day 15 of pregnancy, placental mass and absolute blood flow to placentae were positively correlated $(r=0.34, P<0.01)$.

When examined using ANCOVA, there was a tendency for the number of pups in the same uterine horn to be related to placental mass, although the negative correlation was very weak (Pearson's correlation coefficient $=-0.13, P=0.052$ ). The number of pups in the opposite horn was significantly related to placental mass, although there was only a weak negative correlation (Pearson's correlation coefficient $=-0.23$, $P<0.001)$.

\section{Fetal bodymass}

There was no statistically significant difference in the pattern of fetal mass as a function of both position within a uterine horn and location in the right versus left uterine horns for days 15, 20, 21 and 22 of pregnancy (Fig. 7).

There was a significant $(P<0.01)$ interaction between day of pregnancy and sex of fetus for fetal mass; females were heavier than males on day $20(P<0.05)$, and males were heavier than females on day $21 \quad(P<0.01)$ and day $22(P<0.001)$ of pregnancy (Fig. 8). This sex difference in fetal bodymass is consistent with the rate of either absolute or relative blood flow to the placentae of female versus male fetuses on day 20 and day 22 of pregnancy (Fig. 5), but the data on bodymass and placental blood flow for day 21 do not agree.

The relationship of fetal mass to both absolute blood flow to placentae and placental mass showed some changes over the last week of pregnancy. On day 15 of pregnancy, fetal bodymass was not significantly correlated with either absolute blood flow to placentae $(r=0.17, n=56, p>0.1)$ or with placental mass $(r=0.06, P>0.1)$. On day 20 of pregnancy, fetal bodymass was positively correlated with absolute blood flow to placentae $(r=0.66, n=56, P<0.001)$ but not with placental mass $(r=0.04, P>0.1)$. However, on day 21 of

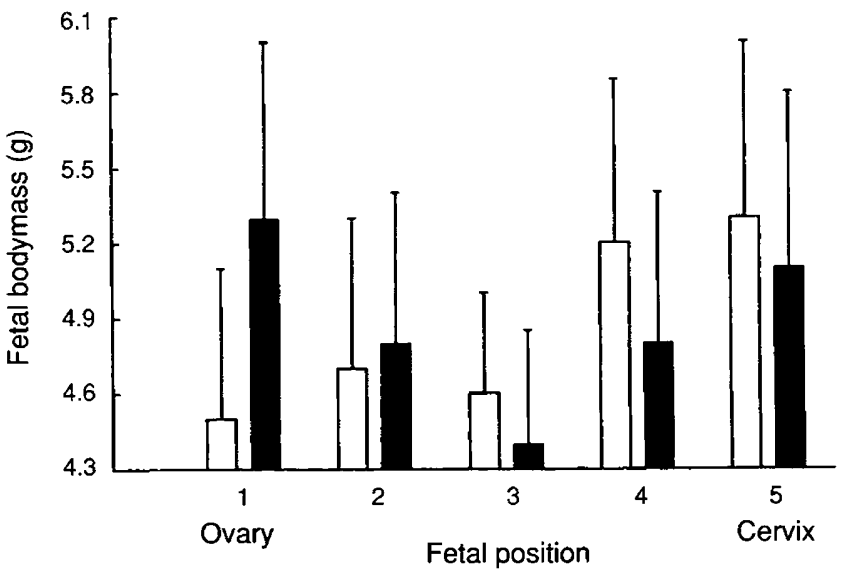

Fig. 7. Mean ( + SEM) bodymass of fetuses located at different positions [groups 1-5: (1) next to the ovary; (2) second position away from the ovary; (3) positions $1-3$ in the middle region of the uterine horn; (4) second position away from the cervix; and (5) next to the cervix] within the left $(\square)$ and right ( $\square$ ) uterine horns on days 15,20 , 21 and 22 of pregnancy in rats.

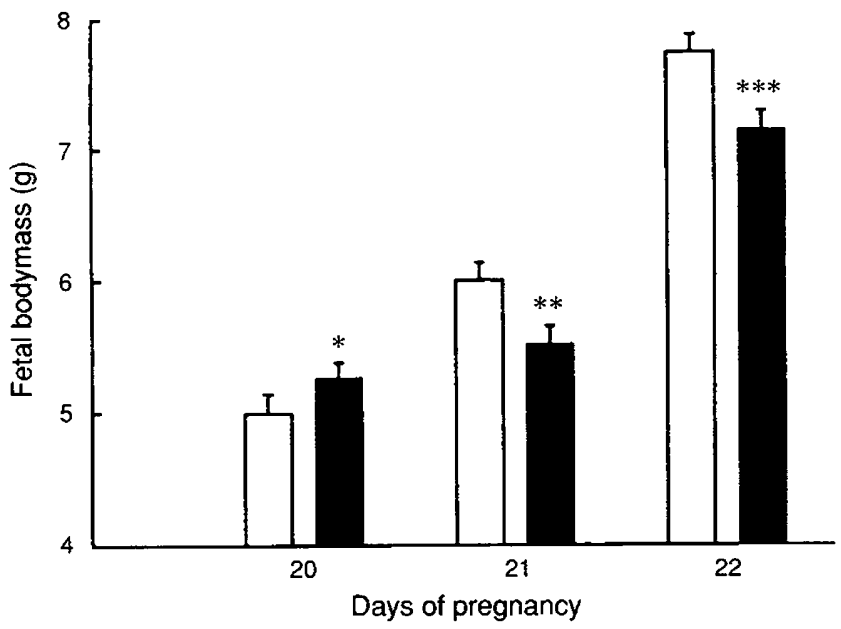

Fig. 8. Mean ( + SEM) bodymass of male $(\square)$ and female $(\boldsymbol{Q})$ fetuses for the last three days of pregnancy in rats. ${ }^{*} P<0.05,{ }^{* *} P<0.01$ and $* * * P<0.001$ indicate significant differences between male and female fetuses.

pregnancy, fetal bodymass and absolute blood flow to placentae were negatively correlated $(r=-0.43, n=62$, $P<0.001$ ), and there was a weak positive correlation between fetal bodymass and placental mass $(r=0.26, n=62, P<0.05)$. Finally, on day 22 of pregnancy, the day of parturition, there was no correlation between fetal bodymass and the absolute blood flow to placentae $(r=0.15, P>0.1)$, while fetal bodymass and placental mass were again positively correlated $(r=0.35, n=64, P<0.01)$. The relationship between fetal bodymass and relative blood flow to placentae was virtually identical to that described for fetal bodymass and absolute blood flow to placentae on days 15,20,21 and 22. ANCOVA showed that the number of pups in the same or opposite horn was not significantly related to fetal bodymass (for data from day 20,21 and 22 of pregnancy; $P>0.1$ ). 


\section{Discussion}

The architecture of the loop uterine artery and vein differed for the right and left uterine horns. For the right uterine horn, the pattern of blood flow to both the uterus and placentae, as well as placental mass and fetal bodymass, all showed the same pattern of having the lowest values in the middle of the uterine horn. All of these findings are consistent with the hypothesis that blood flows into the right uterine horn from both the ovarian and cervical ends, with the lowest rate of flow being in the middle of the right uterine horn.

For the left uterine horn, the results were more complicated. Perhaps because the renal artery serves as the rostral origin of the left uterine loop artery, and not the descending aorta as on the right side, the values for both blood flow to placentae and fetal bodymass were highest at the cervical end and lowest at the ovarian end of the left uterine horn. In contrast, the lowest values for both uterine blood flow and placental mass were in the middle of the left uterine hom and the highest values were at both the ovarian and cervical ends. The reason why these measurements are different in the left uterine horn is unknown. The origin of the uterine vessels from the renal artery and vein in the left uterine horn suggests that the point of lowest rate of blood flow should be shifted toward the ovarian end of the left horn.

The findings concerning uterine blood flow provide indirect support for the hypothesis that blood enters each uterine loop artery at both the ovarian and cervical ends and flows towards the mid-region of each uterine horn. The difference in uterine blood flow to middle compared with end segments of both the right and left uterine horns was most pronounced on day 15 of pregnancy, at which time the rate of blood flow to the ovarian and cervical ends of each uterine horn was over two times greater than to the middle sections. Barr and Brent (1970) found that ligation of the uterine artery in rats at either the ovarian or cervical end did not result in death of all fetuses within the uterine horn, providing additional evidence for the capacity for blood to enter the uterine loop artery from either direction. This possibility was directly confirmed in pregnant mice using an intracardial injection of carbon dye; the dye was visualized entering the uterine loop artery of both the right and left uterine horns from both ends (vom Saal and Dhar, 1992). These findings contradict a prediction made by Meisel and Ward (1981) that blood in the uterine loop artery flows only from the cervical end towards the ovarian end of the uterus in rats.

We found no relationship between the number of fetuses in the same uterine horn and the blood flow to uterine segments, placentae or fetal bodymass. Crowding within a uterine horn decreased the bodymass of fetuses located in the mid-region in mice, which suggested an effect of the number of fetuses in a uterine horn on fetal growth (McLaren and Michie, 1960). It may be that, unlike the putative haemodynamic effects seen by McLaren and Michie (1960) in mice and Eckstein and McKeown (1955) in guinea-pigs, the rat has a method of protecting against the possibility of producing small fetuses in the middle of a uterine horn, as the blood flow to middle uterine segments increased between days 15 and 21 while blood flow to ovarian and cervical segments stayed constant during this time. This hypothesis is supported by the finding that when crowding was induced (an average of nine fetuses) in both the left and right uterine horn in rats by removing one maternal ovary before mating (which results in the remaining ovary ovulating twice the number of ova into the ipsilateral uterine horn; vom Saal et al., 1994), there was no difference in the bodymass of fetuses based on their uterine location ( $K$. Thayer, M. Even and F. vom Saal, unpublished). It has been argued that the type of autoregulation of blood flow to individual placentae that we are suggesting is unlikely (Blizard and Folk, 1990).

A number of studies have examined the relationship between fetal growth and position within the rat uterus (Barr et al., 1970; Ward et al., 1977; Norman and Bruce, 1979a, b). The results of these studies are not consistent with each other or with our findings. The basis for this descrepancy is unknown, although one possibility is that there may be differences among stocks of rats in the architecture of the uterine vessels feeding both the right and left uterine horns. For example, the architecture of the rostral branch of the uterine loop artery feeding both the right and left uterine horns in the rats used in our study was different from that observed by Del Campo and Ginther (1972).

Dzuik (1985) reported that pig fetuses located next to the ovary occupy a greater amount of space than do fetuses located in the middle region of the uterus. He suggested that this could be the basis for the larger bodymass at birth of fetuses located next to the ovary rather than haemodynamic effects. The uterine vasculature in pigs resembles the branching of a tree from a central trunk. Thus, the pattern of blood flow to fetuses (and associated effects on the growth of fetuses in different uterine positions) would presumably be quite different from that in rodents (Oxenreider et al., 1965). We did not attempt to determine whether rat fetuses located at different positions within the uterine horns occupied different amounts of uterine space, although the amount of uterine space available may be less important in rats, which have an invasive, haemochorial placenta, than in pigs, which have a non-invasive, epitheliochorial placenta that occupies a considerable amount of surface area of the uterus.

There was a difference in the rate of blood flow to the placentae of male and female fetuses on day 20 and day 22 of pregnancy, which was similar to the difference in bodymass between sexes on these days; female fetuses weighed more than males on day 20 of pregnancy, while male fetuses weighed more than female fetuses on day 22 . In contrast, on day 21 these measures were significantly negatively correlated: blood flow to the placentae of females was significantly greater than that to males, while the bodymass of males was significantly greater than that of females. The mechanism responsible for the fluctuating rate of blood flow to placentae based on the sex of the fetus supplied and the day of pregnancy, as well as why the relationship between the blood flow to placentae and fetal bodymass changed on each day for the last 3 days of pregnancy, is not known. It is possible that sex steroids mediate these effects.

An unexpected finding was a significant negative correlation between the blood flow to placentae and placental mass on days 20,21 and 22 of pregnancy. Placental mass has typically been assumed to be positively related to placental blood flow and has been used as a predictor of fetal growth potential. However, fetal bodymass was positively correlated with 
placental mass only near the end of pregnancy (day 21 and day 22 , the expected day of parturition), but not on day 20 , confirming previous findings by Norman and Bruce (1979a, b).

We found that blood flow to placentae did not change from day 20 to 22 of pregnancy, while uterine flow dropped markedly between day 21 and day 22 , which is the last day of pregnancy for most rats in our colony. One possible explanation for this finding is that an increase in sympathetic stimulation might selectively act on the uterine vascular supply but not on the vessels feeding the placentae, thus leading to an enhanced vasoconstriction of uterine but not of placental blood vessels on the day of parturition (Greiss and Gobble, 1967). It is also possible that the dramatic decrease in uterine, but not placental, blood flow on day 22 of pregnancy is a passive response resulting from an increase in the tonus of the myometrium that is associated with parturition. This would lead to compression of uterine vessels and an increase in resistance to uterine blood flow. Another factor to be considered is the effect of changes in plasma concentrations of oestradiol and progesterone (Garris, 1988), which occur around parturition in rats (Yoshinga, 1983), on the response of uterine (but not placental) vessels to sympathetic stimulation (Killam et al., 1973; Bell, 1974). Regardless of the mechanisms involved, it is logical to assume that the blood flow to placentae remains relatively constant during parturition so that fetuses continue to be adequately supplied with nutrients and oxygen.

This study was conducted in partial fulfilment of the $\mathrm{PhD}$ thesis by M. D. Even. Support was provided by an NSF Predoctoral Fellowship (RCD-8550750) to M. D. Even, grants from the NSF (DCB-9004806) and the University of Missouri Food for the Twenty-First Century Reproductive Biology Program to F. S, vom Saal, and an NIH grant (HL-36088) to M. H. Laughlin.

\section{References}

Barr M and Brent R (1970) The relation of the uterine vasculature to fetal growth and the intrauterine position effect in rats Teratology 3 251-260

Barr M, Jensh R and Brent R (1969) Fetal weight and intrauterine position in rats. Teratology 2 241-246

Barr M, Jensh R and Brent R (1970) Prenatal growth in the albino rat: effects of number, intrauterine position and resorptions. Journal of Anatomy 128 413-428

Bell C (1974) Control of uterine blood flow in pregnancy Medical Biology 52 219-228

Blizard D and Folk T (1990) Resource sharing in rat gestation: role of maternal cardiovascular hemodynamics American journal of Physiology $\mathbf{2 5 8}$ 1299-1307

Bruce N and Abdul-Karim R (1973) Relationships between fetal weight, placental weight and maternal placental circulation in the rabbit at different stages of gestation Journal of Reproduction and Fertility 32 15-24

Clemens L, Gladue B and Coniglio L (1978) Prenatal endogenous androgenic influences on masculine sexual behavior and genital morphology in male and female rats Hormones Behavior 10 40-53
Del Campo $C$ and Ginther $O$ (1972) Vascular anatomy of the uterus and ovaries and the unilateral luteolytic effect of the uterus: guinea pigs, rats, hamsters, and rabbits American Journal of Veterinary Research 33 2561-2578

Dziuk P (1985) Effect of migration, distribution and spacing of pig embryos on pregnancy and fetal survival. Journal of Reproduction and Fertility Supplement 33 57-63

Eckstein P and McKeown T (1955) Effect of transection of one horn of the guinea-pig's uterus on foetal growth in the other horn Journal of Endocrinology 12 97-107

Eckstein P, McKeown T and Record R (1955) Variation in placental weight according to litter size in the guinea-pig Journal of Endocrinology 12 108-114

Garris DR (1988) Effect of diabetes on uterine conditon, decidualization, vascularization, and corpus luteum function in the pseudopregnant rat Endocrinology $122665-672$

Greiss F and Gobble F (1967) Effect of sympathetic nerve stimulation on the uterine vascular bed American Journal of Obstetrics and Gynecology 97 962-967

Hammond I (1944) Physiological factors affecting birth weight Proceedings Nutrition Society 3 8-12

Healy M, McLaren A and Michie D (1960) Foetal growth in the mouse Proceedings Royal Society $153 \quad 367-379$

Ibsen $H$ (1928) Prenatal growth in guinea-pigs with special reference to environmental factors affecting weight at birth Joumal of Experimental Zoology 51 51-91

Killam A, Rosenfeld C, Battaglia F, Makowski E and Meschia G (1973) Effect of estrogens on the uterine blood flow of oophorectomized ewes American Journal of Obstetrics and Gynecology 115 1045-1052

McLaren A (1965) Genetic and environmental effects on foetal and placental growth in mice Journal of Reproduction and Fertility 9 79-98

McLaren A and Michie D (1959) Superpregnancy in the mouse. I. Implantation and foetal mortality after induced super-ovulation in females of various ages Journal of Experimental Biology 36 281-300

McLaren A and Michie D (1960) Control of pre-natal growth in mammals Nature 187 363-365

Meisel R and Ward I (1981) Fetal female rats are masculinized by male littermates located caudally in the uterus Science 213 239-242

Norjavaara E, Olofsson J, Gäfvels M and Selstam G (1987) Redistribution of ovarian blood flow after injection of hurnan chorionic gonadotropin and luteinizing hormone in the adult pseudopregnant rat Endocrinology $\mathbf{1 2 0}$ 107-114

Norman N and Bruce N (1979a) Fetal and placental weight relationships at days 13 and 17 of gestation Journal of Reproduction and Fertility 57 345-348

Norman $\mathrm{N}$ and Bruce $\mathrm{N}$ (1979b) Fetal and placental weight relationships in the albino rat near term Teratology 19 245-250

Oxenreider S, McClure R and Day B (1965) Arteries and veins of the swine Joumal of Reproduction and Fertility 9 19-27

vom Saal F and Dhar M (1992) Blood flow in uterine loop artery and loop vein is bidirectional in the mouse: implications for transport of steroids between fetuses Physiology and Behavior 52 163-171

vom Saal FS, Finch CE and Nelson JF (1994) Natural history and mechanisms of aging in humans, laboratory rodents and other selected vertebrates. In Physiology of Reproduction 2nd Edn Vol. 2 pp 1213-1314 Eds E Knobil, J Neill and D Pfaff. Raven Press, New York

Ward W, Karp C and Aceto H (1977) Developmental effects of the uterine environment: dependence on fetal sex in rats Journal of Reproduction and Fertility 50 269-274

Yoshinga K (1983) Superimplantation rats as a tool for the study of onset of parturition. In The Anterior Pituitary Gland pp 367-375 Ed. A Bhatnagar. Raven Press, New York 